\title{
IRAS OBSERVATIONS OF NGC 1052
}

\author{
G. NEUGEBAUER AND B. T. SOIFER
}

Division of Physics, Mathematics, and Astronomy, 320-47

California Institute of Technology, Pasadena, California 91125

W. RICE

Jet Propulsion Laboratory, 4800 Oak Grove Drive, Pasadena, California 91109

AND

M. ROWAN-ROBINSON

Mathematics Department, Queen Mary College, Mile End Road, London, E1 4NS, England

Received 9 August 1984

\begin{abstract}
The galaxy NGC 1052 has been observed with the IRAS satellite. The infrared emission at $100 \mu \mathrm{m}$ is substantially larger than a smooth extrapolation of the radio data. Because of the large diaphragm size of IRAS, it is impossible to decide uniquely if the infrared radiation represents a self-absorbed nonthermal spectrum or thermal reradiation by heated dust.

Key words: galaxies-infrared emission
\end{abstract}

\section{IRAS ${ }^{1}$ Observations of NGC 1052}

The elliptical galaxy NGC 1052 is an unusual galaxy known to have an infrared excess at $10 \mu \mathrm{m}$ and $20 \mu \mathrm{m}$ which is too high to be attributed to the general stellar content of elliptical galaxies (Rieke and Low 1972; Rieke, Lebofsky, and Kemp 1982 (henceforth RLK); Becklin, Tokunaga, and Wynn-Williams 1982 (henceforth BTW)). RLK conclude that NGC 1052 contains a blazar (Angel and Stockman 1980) with an exceptionally low luminosity, and argue that the excess infrared emission is due to the blazar. Impey (1984) has described NGC 1052 as archetypical of a class of low-luminosity blazars. On the other hand, BTW contend that the infrared excess in NGC 1052 is the result of thermal emission from dust heated by a nonthermal source.

Both RLK and BTW conclude that the $10 \mu \mathrm{m}$ and 20 $\mu \mathrm{m}$ emission comes from a nuclear core (hereinafter referred to as the core), which BTW show to be $<4^{\prime \prime}$ in diameter. Surrounding this core is an elliptical galaxy which BTW and RLK conclude has the colors of a normal elliptical galaxy. Both BTW and RLK also observe that the core dominates the emission at wavelengths between $\sim 3.5 \mu \mathrm{m}$ and $20 \mu \mathrm{m}$ and that the emission rises steeply, approximating a power law of slope $\alpha \sim-1.5$ to $-2\left(f_{\nu} \propto \nu^{\alpha}\right)$, in the near infrared. Carter et al. (1983) have combined the infrared data of RLK with visual CCD observations and conclude that a power law with

\footnotetext{
${ }^{1}$ The Infrared Astronomical Satellite (IRAS) was developed and operated by the Netherlands Agency for Aerospace Programs (NIVR), the United States National Aeronautics and Space Administration (NASA), and the United Kingdom Science and Engineering Research Council (SERC).
}

slope $\alpha=-1.5$ fits the emission continuum from the core at wavelengths ranging from the visible through 20 $\mu \mathrm{m}$. At radio wavelengths a compact and variable core of diameter 0.'001 is present (Cohen et al. 1971), accompanied by a $30^{\prime \prime}$ double source containing two active regions (Wrobel 1984). A strong emission-line spectrum is seen in the visible from a region $\sim 20^{\prime \prime}$ in diameter (Fosbury et al. 1978). In this paper we present the results of observations from the IRAS survey which extend the infrared measurements to $100 \mu \mathrm{m}$.

\section{Observations}

The IRAS mission and satellite have been described by Neugebauer et al. (1984a). NGC 1052 was observed in eight separate scans forming three hours-confirmed (Neugebauer et al. 1984b) scans. The observations were made in 1983 July 27, August 7, and August 30. NGC 1052 was clearly detected at $25 \mu \mathrm{m}, 60 \mu \mathrm{m}$, and $100 \mu \mathrm{m}$ in the survey with signal-to-noise ratios around 10 in each hours-confirmed set. There was no indication of variability in the observations larger than the $10 \%$ attributable to the systematic photometric inaccuracies.

Coaddition of the scans was necessary to measure the source at $12 \mu \mathrm{m}$; the resultant signal-to-noise ratio at 12 $\mu \mathrm{m}$ after coaddition of all the scans was 11 . The results are given in Table I and plotted in Figure 1. The IRAS flux density at $12 \mu \mathrm{m}$ is well above the fluxes of RLK and BTW, who use diaphragms of 5.'5 and $4^{\prime \prime}$ diameter, respectively. Presumably this is due to the large diaphragms used in IRAS $\left(0.8^{\prime} \times 4.5^{\prime}\right.$ at $12 \mu \mathrm{m}$ and $\left.25 \mu \mathrm{m}\right)$, and the presence of the elliptical galaxy; see below.

The observations have been calibrated using the June 


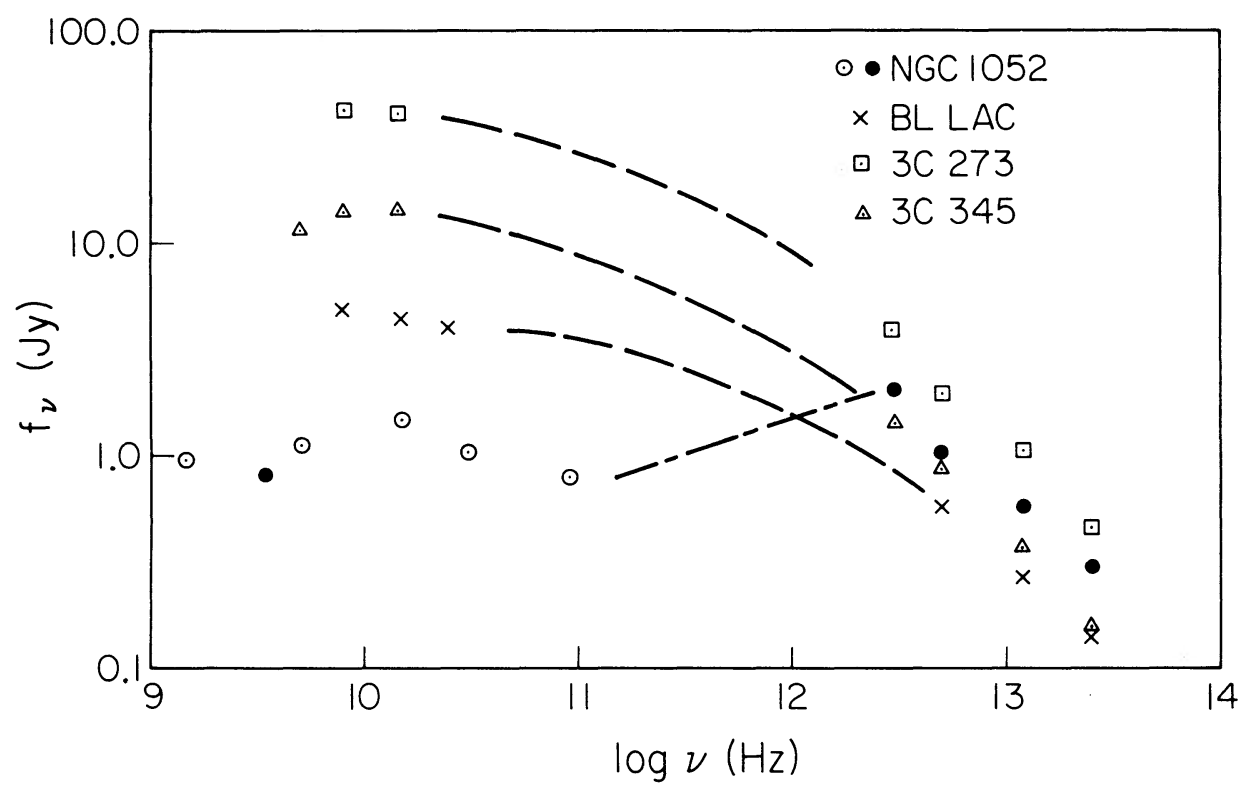

FIG. 1-The energy distributions of NGC 1052 and three well-known radio sources. The infrared observations are all from IRAS. The $100 \mu \mathrm{m}$ observations of BL Lac are confused by infrared "cirrus" (Low et al. 1984) and thus no flux density at $100 \mu \mathrm{m}$ can be quoted. The radio measurements of NGC 1052 indicated with the filled circle were taken by Heeschen (1984) at the same time as the IRAS observations. The open circles represent observations of NGC 1052 made in 1980 and 1981 (Wrobel 1984). The radio observations of BL Lac, 3C 345, and 3C 273 were taken at the same time as the IRAS observations by Aller, Aller, and Haddock (1984).

TABLE I

Infrared Flux Densities (Jy) of NGC 1052

\begin{tabular}{|c|c|c|c|c|}
\hline \multicolumn{5}{|c|}{ wavelength $(\mu \mathrm{m})$} \\
\hline & 12 & 25 & 60 & 100 \\
\hline IRAS & $0.27 \pm 0.03$ & $0.53 \pm 0.08$ & $0.89 \pm 0.06$ & $1.88 \pm 0.07$ \\
\hline \multicolumn{5}{|l|}{ Case $I * *$} \\
\hline core & $0.20 \pm 0.03 *$ & $0.60 \pm 0.10 *$ & 0.9 & 1.9 \\
\hline galaxy & 0.07 & 0.04 & 0.0 & 0.0 \\
\hline \multicolumn{5}{|l|}{ Case II** } \\
\hline core & $0.20 \pm 0.03 *$ & $0.60 \pm 0.10^{*}$ & 0.6 & 0.0 \\
\hline galaxy & 0.07 & 0.04 & 0.3 & 1.9 \\
\hline
\end{tabular}

1984 IRAS absolute calibration (Aumann et al. 1984). The calibration incorporates a refined determination of the nonlinear load resistor curve which results in flux values of the secondary standard NGC 6543 which are $0.93,0.88,0.85$, and 0.89 times the preliminary IRAS calibration of that source (Neugebauer et al. 1984a) at $12,25,60$, and 100 microns. The calibration is probably accurate to $5 \%$ at 12 and 25 microns and $10 \%$ at 60 and 100 microns. The IRAS measurements are broad band and thus need a color correction (Neugebauer et al. 1984a). For these corrections, the intrinsic energy distribution of NGC 1052 was assumed to rise into the infrared with a power law of slope $\alpha=-1$; the change in the color correction if $\alpha=-2$ would be $<5 \%$.

\section{Discussion-Core Versus Galaxian Emission}

In order to interpret the IRAS observations it is neces- sary to separate out that portion of the total flux observed from NGC 1052 (Fig. 1, Table I) which arises in the compact nuclear core from that which arises in the elliptical galaxy which presumably has a stellar-like energy distribution. Although many assumptions about the split between the core emission and the galaxian emission are well within the combined uncertainties of the ground-based and IRAS measurements, we will adopt the hypothesis that at $12 \mu \mathrm{m}$ and $25 \mu \mathrm{m}$ the core contributes the flux densities derived by Carter et al. (1983) from the small-diaphragm ground-based observations. Critical to this assumption is the observation by BTW that the infrared core at $10.6 \mu \mathrm{m}$ is not extended by more than $4^{\prime \prime}$.

The ground-based and IRAS observations can be shown to be consistent with each other. It is first necessary to predict the contribution of the elliptical galaxy outside the core. Observations by Becklin and Neugebauer (1970) show that the $2.2 \mu \mathrm{m}$ flux density of NGC 1052 within a $133^{\prime \prime} 5$ diameter diaphragm is $f_{\nu}(2.2$ $\mu \mathrm{m})=0.18 \pm 0.02 \mathrm{Jy}$. The growth curve for elliptial galaxies at $2.2 \mu \mathrm{m}$ given by Frogel et al. (1978) indicates that the $2.2 \mu \mathrm{m}$ flux density attributable to the galaxy within the $0.8 \times 4.5$ IRAS diaphragm size is consequently $\sim 0.60 \mathrm{Jy}$. The extrapolation of these observations to $12 \mu \mathrm{m}$ and $25 \mu \mathrm{m}$ is uncertain since Impey, Wynn-Williams, and Becklin (1984) have shown that the central regions of many "normal" elliptical galaxies have $f_{\nu}(10 \mu \mathrm{m}) / f_{\nu}(2.2 \mu \mathrm{m})$ as high as $0.22 \pm 0.1$. If this large $f_{\nu}(10 \mu \mathrm{m}) / f_{\nu}(2.2 \mu \mathrm{m})$ ratio holds for the entire galaxy sam- 
pled by the IRAS diaphragm, the contribution of the galaxy to the IRAS observation at $12 \mu \mathrm{m}$ could be as large as $0.13 \mathrm{Jy}$ and the core would contribute only 0.14 $\mathrm{Jy}$, less than the determination of $0.2 \mathrm{Jy}$ by Carter et al. (1983). Thus the high $f_{\nu}(10 \mu \mathrm{m}) / f_{\nu}(2.2 \mu \mathrm{m})$ ratio presumably does not hold for the entire elliptical galaxy surrounding the core of NGC 1052. If, on the other hand, the energy distribution of the galaxian component of NGC 1052 were to follow that of a hot blackbody between $2.2 \mu \mathrm{m}$ and $12 \mu \mathrm{m}$ the flux density at $12 \mu \mathrm{m}$ of the galaxy would be $f_{\nu}(12 \mu \mathrm{m}) \sim 0.02 \mathrm{Jy}$. Thus the value implied if the core emits $0.2 \mathrm{Jy}$ at $12 \mu \mathrm{m}$, namely $0.07 \mathrm{Jy}$ at $12 \mu \mathrm{m}$ (see Table I), lies well within the range of possibilities.

The emission from the core at $25 \mu \mathrm{m}$ must be determined by an extrapolation from the ground-based measurements at $20 \mu \mathrm{m}$. If the power-law slope is $\alpha=-1.5$ the flux density at $25 \mu \mathrm{m}$ contributed by the core alone would be $0.6 \mathrm{Jy}$, larger than the total measured with IRAS. The observations, then, if taken at face value, would imply no or little contribution from the galaxy at $25 \mu \mathrm{m}$. The uncertainties in the combined measurements and in the extrapolations, however, easily encompass the $25 \mu \mathrm{m}$ flux density at $0.04 \mathrm{Jy}$ (see below and Table II) predicted from a normal elliptical galaxy whose flux density at $12 \mu \mathrm{m}$ is $0.07 \mathrm{Jy}$. Changing the assumed power law slope of the energy distribution of the core to $\alpha=$ -1 , consistent with the longer wavelength IRAS observations, decreases the amount attributable to the core at $25 \mu \mathrm{m}$ and would clearly make the fit better.

In order to estimate the flux densities of the core at 60 $\mu \mathrm{m}$ and $100 \mu \mathrm{m}$, it is again necessary to estimate the flux densities of the galaxy surrounding the core. Unfortunately, few measurements of elliptical galaxies at the longer IRAS wavelengths have been analyzed at this time. A preliminary search for infrared emission associated with galaxies in the Revised Shapley-Ames Catalog of Bright Galaxies (Sandage and Tammann 1981) showed a complete absence of elliptical galaxies at the IRAS wavelengths (deJong et al. 1984a). Furthermore, a preliminary study of many elliptical galaxies in the IRAS data has not shown significant $100 \mu \mathrm{m}$ emission except in those cases when visual observations would have given evidence for dust or gas (deJong 1984b). In this regard, it should be noted that Carter et al. (1983) show the presence of a weak diffuse dust lane extending $\sim 20^{\prime \prime}$ along the minor axis of NGC 1052.

TABLE II

$\begin{array}{lccc}\text { Infrared Flux Density } & \text { Ratios of E1liptical Galaxies } \\ & \text { M31 (nuc) } & \text { M32 } & \text { NGC } 205 \\ \mathrm{f}_{\nu}(25 \mu \mathrm{m}) / \mathrm{f}_{\nu}(12 \mu \mathrm{m}) & 0.54 & 0.58 & 0.68 \\ \mathrm{f}_{\nu}(60 \mu \mathrm{m}) / \mathrm{f}_{\nu}(12 \mu \mathrm{m}) & 5.1 & <0.9 & 4.8 \\ \mathrm{f}_{\nu}(100 \mu \mathrm{m}) / \mathrm{f}_{\nu}(12 \mu \mathrm{m}) & 25 & <4.1 & 34\end{array}$

Some elliptical galaxies do show significant $100 \mu \mathrm{m}$ emission, however. Table II gives the observed flux density ratios at IRAS wavelengths of M32, NGC 205, and the nucleus of M31 where there is evidence that little gas and dust is present (Habing et al. 1984, revised). In each case, $f_{\nu}(25 \mu \mathrm{m}) / f_{\nu}(12 \mu \mathrm{m}) \sim 0.6$. It is clear from Table II, however, that these apparently old stellar systems show a wide range in their far infrared properties. NGC 205 is also known from optical and radio studies to contain significant amounts of gas and dust.

Although there is no evidence from visible images for large amounts of dust in the elliptical galaxy associated with NGC 1052, it is necessary to consider two extreme cases when examining the nature of the core. In the first case (case I of Table I), the elliptical galaxy contributes negligible flux at $60 \mu \mathrm{m}$ and $100 \mu \mathrm{m}$, and the observed flux densities- $0.9 \mathrm{Jy}$ and $1.9 \mathrm{Jy}-$ represent the core. In the second case (case II of Table I), the galaxy outside the core has an energy distribution like NGC 205 and dominates the observed emission at far infrared wavelength. Clearly, any case in between these two cases is possible. In fact, the ratio $f_{\nu}(100 \mu \mathrm{m}) / f_{\nu}(12 \mu \mathrm{m})$ cannot be as extreme for the galaxian component in NGC 1052 as it is in NGC 205 if the galaxy indeed contributes 0.07 Jy to the IRAS observations at $12 \mu \mathrm{m}$.

\section{Discussion-Nature of the Core}

For comparison, Figure 1 includes the radio and infrared flux densities of three well-known radio sources3C 273, 3C 345, and BL Lacertae; the latter two are representative blazars. The radio observations were made contemporaneously with the IRAS observations. The observed infrared continua of all four sources in Figure 1 are amazingly similar, with slopes $\alpha \sim-1$. The infrared continua of BL Lac, 3C 345, and 3C 273 form a smooth extension of the radio observations; in the case of blazars at least, this is almost certainly associated with synchrotron emission characteristic of blazar emission in this wavelength region.

The observed energy distribution of NGC 1052 is distinguished from those of the other sources in Figure 1 and from that of the canonical blazars by the fact that the infrared continuum of NGC 1052 falls well above the extrapolation of the radio flux $(1.9 \mathrm{Jy}$ vs. $<0.8 \mathrm{Jy}$ ). (In this respect the observed energy distribution resembles a less extreme example of those of the radio-quiet quasars observed by Neugebauer et al. (1984b).) If the Hubble constant is taken as $75 \mathrm{~km} \mathrm{~s}^{-1} \mathrm{Mpc}^{-1}$, NGC 1052 is at a distance of $19 \mathrm{Mpc}$, implying a total luminosity for the assumed far-infrared emission from the core of $5 \times 10^{8}$ $L_{\odot}$. This luminosity is comparable to the luminosity between $5 \mu \mathrm{m}$ and $20 \mu \mathrm{m}$ and that of the stellar component in the core as deduced from the 1-3 $\mu \mathrm{m}$ emission (BTW).

RLK postulate that the nonthermal emission from the 
core of NGC 1052 extends from the radio through the infrared at $20 \mu \mathrm{m}$ and $10 \mu \mathrm{m}$. If this is true, a mechanism, presumably self-absorption, is needed to attenuate the flux density by more than a factor of 2 between $100 \mu \mathrm{m}$ and $9 \mathrm{~cm}$, i.e., to provide a continuum with slope $\alpha>$ 0.2. Such a mechanism is needed whether the core or the galaxy emission dominates the IRAS measurements (case I or II), although the qualitative requirements are slightly different. For a homogeneous synchrotron source which is self-absorbed, the spectral index is 2.5 , so that this mechanism is consistent with the observations. Alternatively, Marscher (1977) has shown that if the radiating source is inhomogeneous, the self-absorbed synchrotron continuum can exhibit an optically thick spectral index between 0.5 and 1.5. Although these mechanisms are both sufficient to account for the observed drop-off, the source would be unique among known blazars in having the absorption occur at such a high frequency. In particular, there is no evidence for the rapid variability expected for a source with a turn-off frequency at $60 \mu \mathrm{m}$.

If the infrared continuum is assumed to be due to thermal emission from heated dust, the conditions in the source depend strongly on the assumption as to the division between core and galaxy. If the observed $60 \mu \mathrm{m}$ and $100 \mu \mathrm{m}$ emission represents the core (case I, Table I) the color temperature implied by the $60 \mu \mathrm{m}$ and $100 \mu \mathrm{m}$ fluxes is $T_{\mathrm{c}}=42 \mathrm{~K}$. If the physical temperature of the radiating material equals the color temperature, the total source must subtend an angular diameter larger than 0.22 or $22 \mathrm{pc}$. If the source consists of a shell of 22 pc diameter surrounding a central luminosity source of $5 \times 10^{8}$ $\mathrm{L}_{\odot}$, the equilibrium temperature would be $31 \mathrm{~K}$. Emissivity variations observed in the Galaxy such, as emissivity proportional frequency ${ }^{-1}$ could easily bring the color and equilibrium temperatures into agreement.

If the $60 \mu \mathrm{m}$ and $100 \mu \mathrm{m}$ flux densities observed by IRAS are dominated by the galaxy, the details of the core energy distribution are quite arbitrary. In the case shown in Table I, if the infrared is thermal emission the color temperature derived from the observed $25 \mu \mathrm{m}$ and $60 \mu \mathrm{m}$ flux densities is $150 \mathrm{~K}$, implying the dust grains are located close to the luminosity source. Again this interpretation is consistent with the observations.

\section{Conclusions}

The IRAS measurements, because of their big beam size, cannot uniquely decide whether the infrared emission represents the high-frequency tail of the radio emission or if it represents a thermal component in the nucleus of NGC 1052. The choice is largely one of preference and intuition at this point. The ad hoc assumptions necessary to continue the radio continuum into the infrared persuade us, however, that it is more reasonable to explain the observed emission by thermal radiation from dust heated by a nonthermal source, thus supporting the suggestion by BTW. It should be emphasized that this does not preclude the radio source being a mini-blazar as evidenced by its variability and continuum shape. The IRAS observations give evidence for the presence of thermally heated dust in this galaxy system which dominates the emission in the far infrared.

We thank all our colleagues on the IRAS team for making the mission a success. In particular, we thank E. Kopan and C. Oken for their help in reducing the observations. D. Heeschen, H. and M. Aller, and T. Haddock kindly provided us with concurrent radio measurements of NGC 1052 and of the radio sources. Conversations with C. Impey were most helpful as well as discussions with T. deJong, H. Habing, J. Wrobel, and G. WynnWilliams.

\section{REFERENCES}

Aller, H., Aller, M., and Haddock, T. 1984 (personal communication). Angel, J. R. P., and Stockman, H. S. 1980, Ann. Rev. Astr. Ap. 18, 321. Aumann, H. H., Gillett, F. J., Low, F. G., Neugebauer, G. X., Walker, R. L., Waters, R. 1984 (in preparation).

Becklin, E. E., and Neugebauer, G. 1970, unpublished CIT data.

Becklin, E. E, Tokunaga, A. T., and Wynn-Williams, C. G. 1982, Ap. J. 263, 624.

Carter, D., Jorden, P. R., Thorne, D. J., Wall, J. V., and Straede, J. C. 1983, M.N.R.A.S. 205, 377.

Cohen, M. H., Cannon, W., Purcell, G. H., Shaffer, D. B., Broderick, J. J., Kellerman, K. I., and Jauncey, D. L. 1971, Ap. J. 170, 207.

deJong, T., et al. 1984a, Ap. J. (Letters) 278, L67.

de Jong, T. et al. $1984 b$ (personal communication).

Fosbury, R. A. E., Mebold, V., Goss, W. M., Dopita, M. A. 1978, M.N.R.A.S. 183, 549 ,

Frogel, J. A., Persson, S. E., Aaronson, M., Matthews, K. 1978, Ap. J. $220,442$.

Habing, H. et al. 1984, Ap. J. (Letters) 278, L59.

Heeschen, D. 1984 (personal communication).

Impey, C. 1984 (personal communication).

Impey, C., Wynn-Williams, G., and Becklin, E. 1984 (in preparation).

Low, F. J. et al. 1984, Ap. J. (Letters) 278, L19.

Marscher, A. P. 1977, Ap. J. 216, 244.

Neugebauer, G. et al. 1984a, Ap. J. (Letters) 278, Ll.

Neugebauer, G. et al. 1984b, Ap. J. (Letters) 278, L83.

Rieke, G. H., and Low, F. J. 1972, Ap. J. (Letters) 176, L95.

Rieke, G. H., Lebofsky, M. J., and Kemp, J. C. 1982, Ap. J. (Letters) 252, L53.

Sandage, A. R., and Tammann, G. A. 1981, A Revised Shapley-Ames Catalog of Bright Galaxies (Washington, D.C.: Carnegie Institution of Washington), Publication 635 .

Wrobel, J. M. 1984, Ap. J. 284, 531. 